\title{
BREAST CANCER IN PATIENTS WITH BRCA MUTATION: CASE SERIES
}

\author{
Sabas Carlos Vieira', Danilo Rafael da Silva Fontinele² \\ ${ }^{1}$ Oncocenter; Oncobem - Teresina (PI), Brazil. \\ Universidade Estadual do Piauí - Teresina (PI), Brazil.
}

Introduction: Women who inherit a deleterious mutation in BRCA face substantially increased risks of developing breast cancer, estimated in $70 \%$. Even though the annual screening with magnetic resonance and mammography promotes the early detection of the disease, the gold standard for the primary prevention of breast cancer is still bilateral mastectomy. Objectives: To characterize breast cancer with mutation in the BRCA gene. Method: This is a retrospective study, case series. We included all patients who had tested positive for BRCA mutation and who had a previous or current diagnosis of breast cancer from 1999 to 2019. The study was approved by the Research Ethics Committee of Universidade Federal do Piauí, report n. 2.817.502. Results: We found 10 patients with previous breast cancer, or at the time when they had a mutation in the BRCA gene. They were all female, with mean age of 39.4 years at diagnosis, average of 3.6 cases of cancer in the family. Five (50.0\%) were triple negative tumors, mean size of $6.7 \mathrm{~cm}, 9$ invasive carcinomas, 2 stage IIIB, 3 cases with invasion and impairment of axillary lymph nodes. Three underwent bilateral salpingo-oophorectomy, and 6 underwent bilateral or contralateral risk-reducing mastectomy procedures. All patients were submitted to chemotherapy, being 6 neadjuvant and 5 associated with radiotherapy. As to type of mutation, 6 presented mutation for BRCA1, and 4 for BRCA2. Two patients died because of the disseminated disease. Only one patient is still being followed-up, after 29 months, with annual mammography, resonance and clinical examination, and did not accept risk-reducing mastectomy or salpingo-oophorectomy. Conclusions: The mean age of breast cancer diagnosis was 39.4 years. Half of the patients were triple negative, and most had mutation in BRCA1. Two patients died because of the disseminated disease. 